\title{
A Metal Cylindrical Shell Expansion Loading Technique based on Rock Triaxial Testing System
}

\author{
Shao-Xing ZHANG ${ }^{1, a}$, Xiang-Yu $\mathrm{LI}^{1, \mathrm{~b},{ }^{*},}$, Zhen-Yu ZHANG ${ }^{1, \mathrm{c}}$ and Fang-Yun LU ${ }^{1, \mathrm{~d}}$ \\ ${ }^{1}$ College of Science, National University of Defense Technology, Changsha 410073, China \\ asxzhang10@163.com, ’xiangyu_lee@163.com \\ ${ }^{*}$ Corresponding author
}

Keywords: metal cylindrical shell, quasi-static loading, structural strength, triaxial testing system

\begin{abstract}
This paper presents an expansion loading technique for metal cylindrical shell to effectively achieve a one-dimensional, high-strength and quasi-static tensile loading on shell sidewall. A quasi-static axial compression is firstly loaded on the filling polymer via rock triaxial testing system (RTTS), which results in the internal pressure of filling polymer reaching up to the scale of $100 \mathrm{MPa}$ or even GPa. Such intensive pressure would give rise to a fierce radial expanding of the filling polymer and then consequently generate a uniform quasi-static radial loading on the inner surface of the shell, which provides a one-dimensional high-intensity tensile strength radially. Accordingly, the axial pressure-displacement curve could be recorded by RTTS to characterize the mechanical structural strength of cylindrical shells. This simple and efficient loading technique has been successfully applied to the measurement of the structural strength for Duralumin LY-12.
\end{abstract}

\section{Introduction}

The fracture of Metal shell under high pressure loading is of primary significance in the fields of weapon research and engineering practice, such as the rupture of oil and gas pipelines or high-pressure vessels, fracture on the shells of missiles and warhead, radiation embrittlement of the protective layer in nuclear power station, strength assessment of shell structures in the buildings. All of them are closely correlated to dynamic and static fracture properties of the structure, so the fracture behavior of the metal shells has aroused extensive research.

Expanding pipes or loops techniques are most universally used in the experimental study of dynamic fracture properties of the metal shells [1, 2]. They are generally classified into explosion driven expanding ring (EDER) technique or electromagnetic driven expanding ring (EMDER) technique, according to the experimental device. In the early 1940s, N.F.Mott, G.I.Taylor and R.Gurney had conducted a deep research on the fracture properties of metal shells under inside outward detonation loading and proposed Mott debris distribution formula [3], Taylor fracture model [4] and Gurney empirical formula of fragments initial velocity [5], respectively. Then, D.Grady [6] summarized and further promoted relevant research in this area. The first set of explosive expanding drive ring device was designed by Johnson [7] in 1963. By detonating C3 explosives in the metal cylinder, the specimens attached to the outside are accelerated to a high speed instantaneously, then expand freely, finally break. This method was made important improvements by Later Hoggatt [8], Tiegang Tang $[9,10,11]$ and other scholars. EMDER is firstly proposed by Niordson [12] when he designed the first set of electromagnetic drive expanding ring in 1965. When the solenoid is provided with instantaneous current, due to electromagnetic induction, another current would be formed in metal ring sample, so the ring would experience radial expansion. Then Grady [13] improve the defects in the original circuit, electromagnetic coil and sample preparation. Gui [14] solved two key issues: electromagnetic discharge and short-circuit switch, thus achieving free expansion.

In the area of static expansion loading on metal shell, loading by high pressure water is now widely used in sealed container. However, this way requires super-high sealing, and is difficult to achieve a break-level stretching in the case of a small container. Thus, under laboratory conditions, the static tensile properties of thin metal plate call for further study. Wenfu Xu [15] used universal tensile 
machine, Shimadzu AG-10TA, to test plate tensile, notched tensile, shear tensile of Aluminum Alloy 6063 and researched deformation and damage behaviors of the aluminum alloy at three different stress states. Rui Cao [16] studied the fracture process and fracture mechanism of TiAl Alloy through a situ tensile test on TiAl alloy sample plate with different notch.

Triaxial testing machine is commonly used to test mechanical properties of rock, metal and other materials. The world's first test machine, relying on a lever device, was built about 300 years ago in Leiden University by professor Musschenbroeck. In 1962, Turner and Barnard developed a testing machine with a rigid frame, a large fuel tank and low height, which can product a load-independent constant strain rate and measure the complete stress-strain curve of the material. In the early 1970s, electro-hydraulic servo testing machine was successfully developed. Automatic control technique using mechanical operation based on pulse feedback principle can record displacement, velocity, load and rate of loading relying on feedback system, so that we can get stress-strain curve of rock specimen. At present, advanced triaxial testing systems in laboratory are all the use of this principle $[17,18]$. After a long period of development, a fully functional, simply operational, highly precise modern triaxial testing system has been gradually formed and widely used in the research and applications of geotechnical engineering, construction materials, geological disaster and so on.

To conclude, in the research of material's dynamic breaking properties under high strain rate loading, EDER and EMDER techniques are recognized as the most effective experimental techniques[19,20]. However, the loading product and loading effect may have an undesirable influence on the performance of samples and simultaneously, the samples' expansion, strain and stress cannot be accurately controlled and measured. For static loading, the gigantic hydraulic equipment and experimental device have a stringent requirement for sealing, or the water and oil would leaked, which makes it difficult to test and recycle samples. In the flat tensile test, the clamping, designing and processing for sample very complex. To achieve the static high pressure on metal cylindrical shell measure the structural properties for metal samples more effectively, a convenient expanding loading technique has been designed.

\section{Experimental Section}

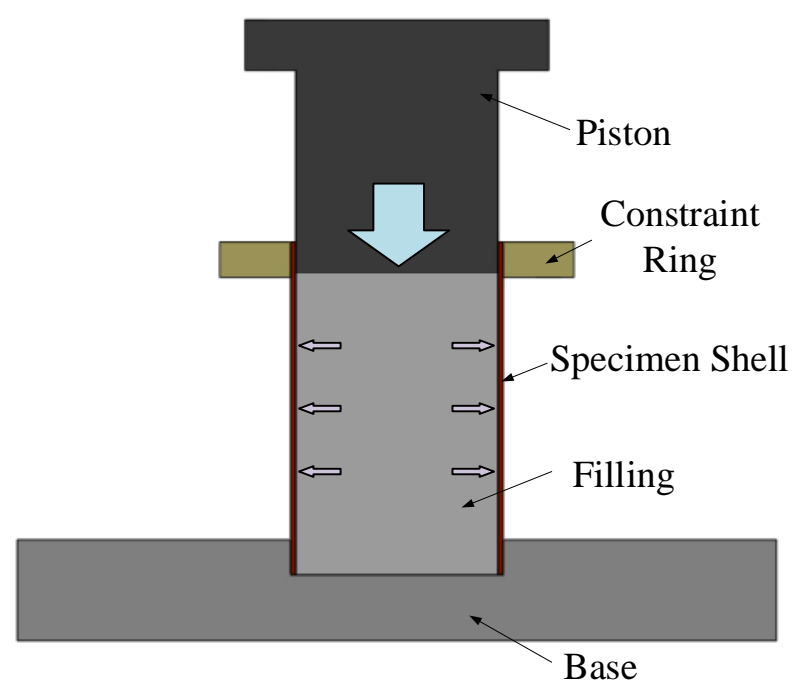

Fig.1 Cross-sectional view of the experiment

The setup of the experiment is schematically shown in Fig.1. Experimental devices include a base, a filler, a specimen shell, a constraint ring and a piston. The base, constraint ring and piston are all composed with steel with great hardness. The base, put on the working platform of the triaxial testing machine which moves up with a constant speed, and the constraint ring are fixed at opposite ends of the specimen case. The polymeric filler is placed inside the specimen shell. When the top of the piston touches the pressure sensor, the filler was driven to produce axial strain. Duo to the Poisson 
Effect, the filler expands and applies uniform radial pressure on the shell. The specimen shell starts to deform elastically and then plastically until a rupture happens on the shell. The pressure-displacement curves then could be measured by the pressure sensor touching the piston.

The experimental devices are shown in Fig. 2. Filler material is made from PTFE, and the piston, constraint ring and base are constituted of Steel 45\#. Specimen shells are Duralumin LY-12 and their sizes are displayed in Tab.1. Specimen from No.1 to 4 are preset a "V" trough angled $60^{\circ}$ with different depths along the generatrix in the out surface of the shell. Specimen No.5 has no trough which is used in a check experiment. TAW-2000 electro-hydraulic servo triaxial testing system is used in this experiment to record the pressure-displacement curve. Results measured are shown in Fig.3, in which data tagged with "No Shell" is got from the control experiment. Fig. (b) is the partial enlarged view of Fig.(a).

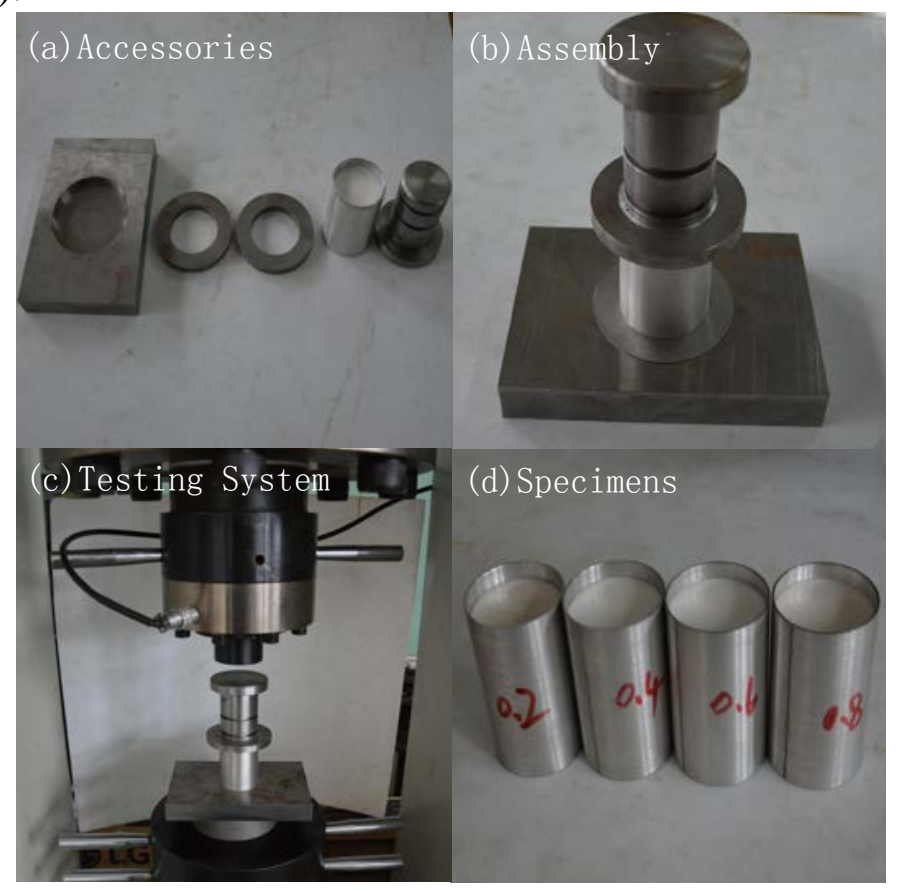

Fig.2 Components of axial loading expanding shell system

Table 1. Parameters of the specimen shell

\begin{tabular}{ccccc}
\hline Test No. & $\begin{array}{c}\text { Interior } \\
\text { Diameter/mm }\end{array}$ & $\begin{array}{c}\text { Exterior } \\
\text { Diameter/mm }\end{array}$ & Height/mm & $\begin{array}{c}\text { Depth of } \\
\text { Trough/mm }\end{array}$ \\
\hline No.1 & 40 & 42 & 66 & 0.2 \\
No.2 & 40 & 42 & 66 & 0.4 \\
No.3 & 40 & 42 & 66 & 0.6 \\
No.4 & 40 & 42 & 66 & 0.8 \\
No.5 & 40 & 42 & 66 & 0 \\
\hline
\end{tabular}

\section{Results and Discussion}

From Fig. 3, it is obvious that the expanding of specimen No.5 is composed of three stages. In the first stage from Point A to Point B, the filler expands freely since there is a certain gap between PTFE filler and shell, at which period nothing noticeable happens. Compared with the "No Shell" curve, it is found that the PTFE filler is in the phase of elastic deformation, because the slope of pressure-displacement curve is constant. At the end of this phase, the slope starts to change, which means that the filler begins to deform plastically. In the second phase between Point B and Point C, the filler touches the shell closely and the shell is driven to expand. The filler deforms plastically 
while the specimen is in the elastic stage in this phase. In the third stage, after Point $\mathrm{C}$, the curve becomes gentler and keeps a certain slope, indicating that the shell is in the stage of deformation which maintains a long period. At Point $\mathrm{D}$, where axial displacement reaches $8.78 \mathrm{~mm}$, the shell fracture occurs.
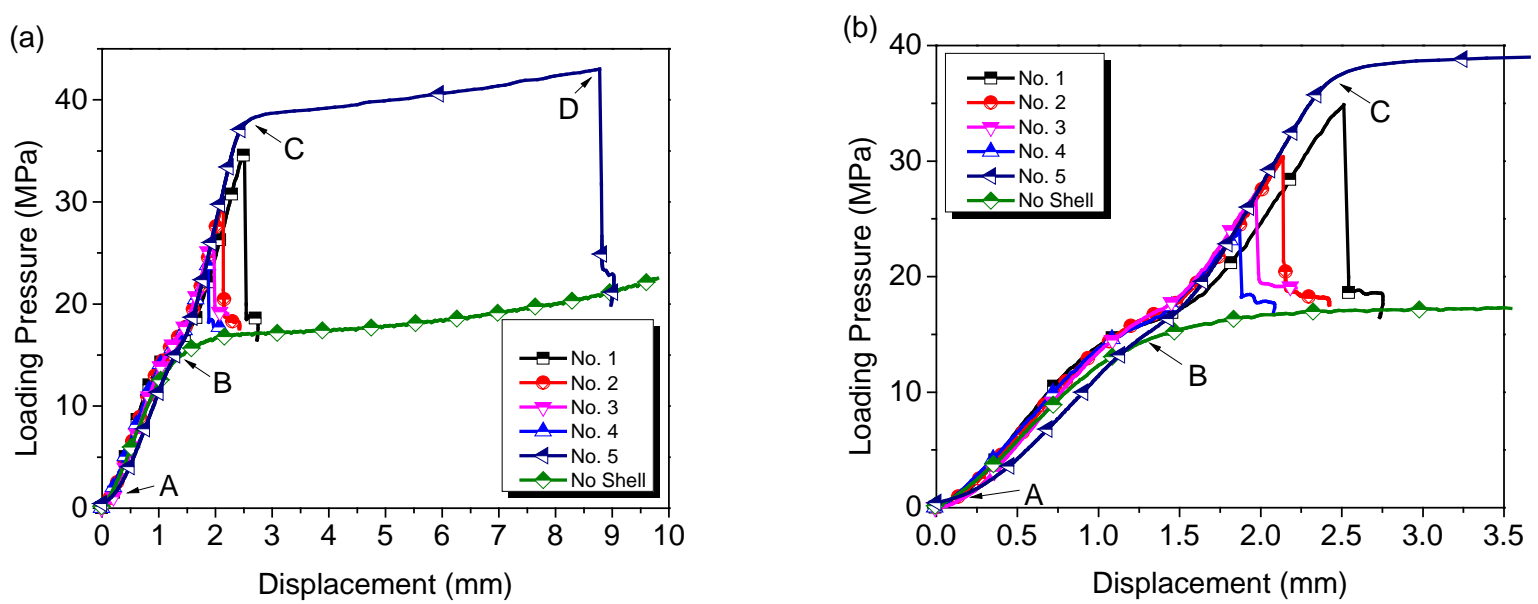

Fig.3 Loading pressure of Duralumin LY-12 expanding shell: A. Commencement of axial pressure;

B. Exordial loading on specimen; C. Transition from elastic deformation to plastic deformation;

D. Fracture point of specimen

Expanding process of specimen from No.1 to 4 has only two phases. The first phase is the same as that of specimen No.5. In the second stage, the shell is still experiencing elastic deformation, while there is a significant stress concentration at the preset trough. At this position, shell deforms plastically and fractures in a second. The curves of different tests remain consistent well in this stage, which indicates the stability and reliability of this newly proposed experimental technique.

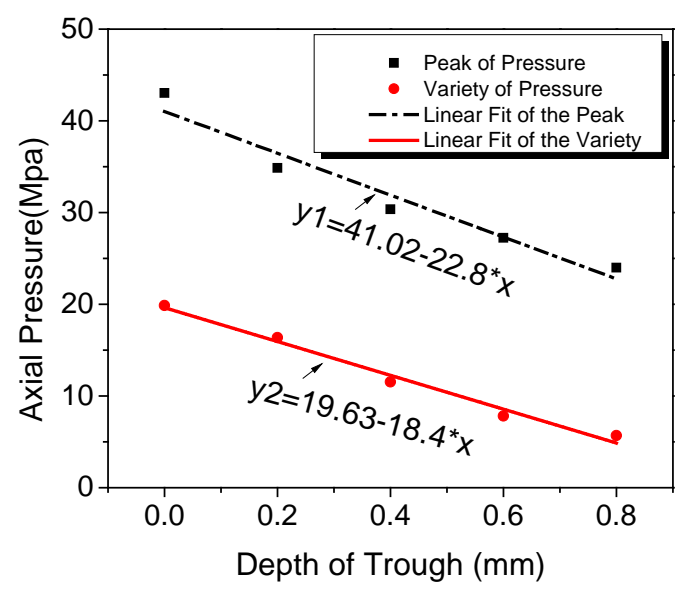

Fig.4 Peak and variety of pressure when specimens rupture

Both the peak pressure of the curve and the variety of the pressure when fracture happens are important indicators of the mechanical structure strength of the specimen shell. These two data are displayed in the Fig.4. It shows a good linear correlation between mechanical strength and depth of trough. In another word, with the increasing of trough's depth, mechanical strength of the shell linearly slips downward. It is fairly consistent with the theoretical analysis.

After static loading, on the specimen shell, it is found that stress concentration occurs at the angle of $45^{\circ}$ to both sides of the bottom of the trough, where metal color is different from other part. The fracture occurred at the $45^{\circ}$ direction, which is the typical shear fracture, as shown in Fig.5. 


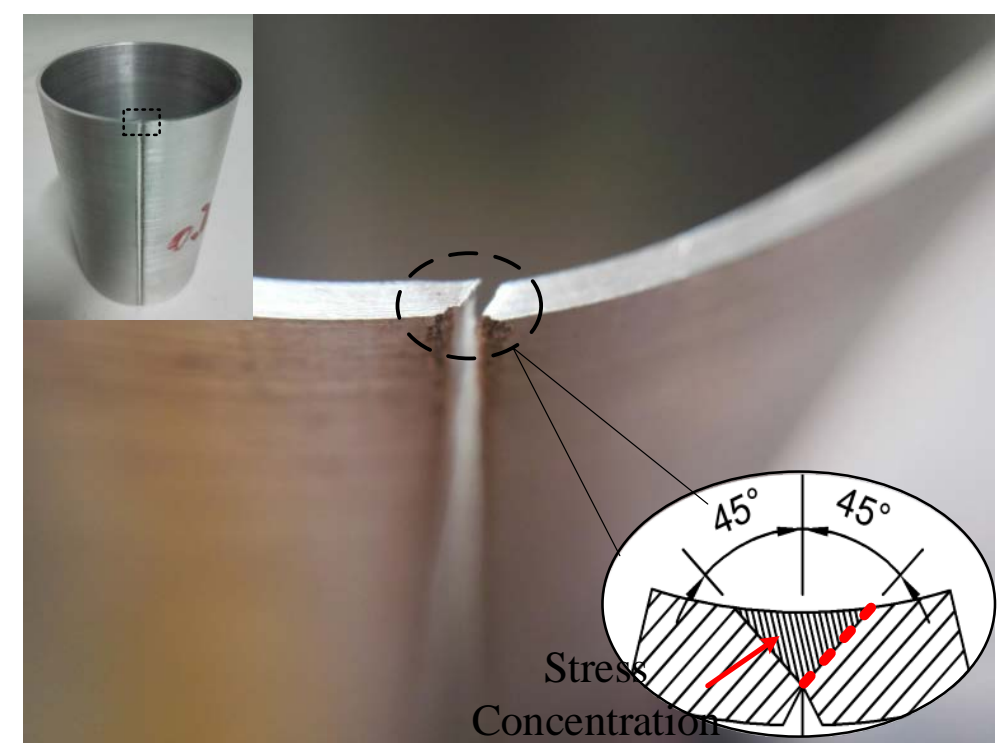

Fig.5 The rupture of $0.2 \mathrm{~mm}$ trough expanding shell

As shown in Fig. 6, the tensile fracture of specimen No.5 also happens at $45^{\circ}$. There is a clear slight depression on the surface of the shell. It indicates that the necking happened in this process. On the other hand, specimen No.1-4 have a smooth surface which evident the conclusion that specimen with no groove is plastically deformed while specimen with groove is elastically deformed in general. That's why the no-notch specimen's deformation is much larger than those with groove. The device can continue to provide a uniform static high pressure loading, which proves the effectiveness of the experimental technique.
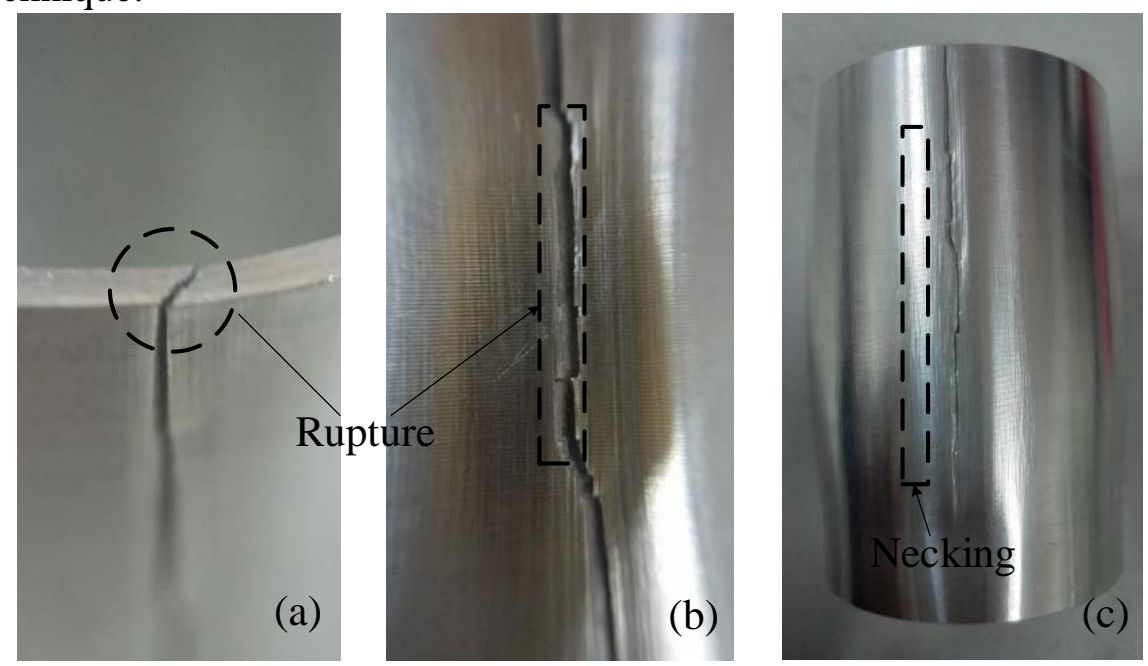

Fig.6 The rupture of no trough expanding shell: (a) Cross section of rupture; (b) Fissure of rupture;

(c) Texture of Necking

\section{Summary}

In this paper, a convenient, economical and effective expansion loading technique for studying the static tensile properties and fracture characteristics of shell materials is presented. This technique, which provides a controllable radial high static pressure on a metal cylindrical shell by compressing polymeric filler, is different from other dynamic expanding loop loading technique. Using this brand-new technique, structural strength and fracture characteristics of Duralumin LY-12 have been gained. 


\section{Acknowledgement}

The authors would like to acknowledge financial supported by the China National Natural Science Founding (11202237\&11132012).

\section{References}

[1] Warnes R H, Duffey T A, Karpp R R, et al. An improved technique for determining dynamic material properties using the expanding ring, M. Meyers M A, Murr L E. Shock Waves and High-strain-rate Phenomena in Metals. New York: Plenum Press, 1981, pp. 23-36.

[2] TGang Tang, Gu Yan, QZ Li, J. Explosion and Shock, 2003, 23(6) 529-533.

[3] Mott N F. A theory of fragmentation of shells and bombs, R. 1943.

[4] Taylor G I. The fragmentation of tubular bombs, C. Batchelor G K. The Scientific Papers of Sir Geoffrey Ingram Taylor: Vol. 3. Cambridge: Cambridge University Press, 1963, pp. 387-390.

[5] Gurney R W. The initial velocities of fragments from bombs, shell, grenades, R. BRL Report No. 405, 1943.

[6] Grady D. Fragmentation of rings and shells, M. Berlin: Springer Science + Business Media Deutschland GmbH (2006).

[7] Johnson P., Stein B., Davis, R. Measurement of dynamic plastic flow properties under uniform stress, C. Symposium on the Dynamic Behavior of Materials, Conference Location, 1963, pp.195-198.

[8] Hoggatt C R, Recht R F, J. Experimental Mechanics, 1969, 6(10) 441-448.

[9] TGang Tang, QZ Li, YongTao Chen, Gu Yan, ChangLi Liu, J. Explosion and Shock, 2009, 29 (5) 546-549.

[10] TGang Tang, QZ Li, J. Explosion and Shock, 2009, 29 (6) 561-565.

[11] TGang Tang, YuLin Gui, QZ Li, J. Explosion and Shock, 2011, 30 (5) 501-510.

[12] Niordson, F. I, J. Experimental Mechanics, 1965, 5 (1) 29-23.

[13] Grady D., Benson D, J. Experimental Mechanics, 1983, 23 (4) 393-400.

[14] YULin Gui, CWei Sun, Qiang Li, J. Explosion and Shock, 2007, 26 (6) 481-485.

[15] WenFu Xu, HYan Che, JianH Chen, J, Mechanical Engineering, 2009, 33 (1) 20-22.

[16] Rui Cao, YouZi Lin, JianH Chen, J, Mechanical Engineering, 2008, 44 (1) 40-45.

[17] XueChao Niu, QXi Zhang, ZWen Yue, J. Rock Mechanics, 2013, 34 (2) 600-607.

[18] XueChao Niu, QXi Zhang, ZWen Yue, J, Coal Science and Technology, 2012, 40 (4) 1-5.

[19] Janiszewski J, J. International Journal of Solids and Structures, 2012.

[20] Vadillo G., RodrígueZ J., Fernández-Sáez J. On the interplay between strain rate and strain rate sensitivity on flow localization in the dynamic expansion of ductile rings, J. International Journal of Solids and Structures, 2011. 